\title{
THERAPEUTIC EFFICACY OF VITAMIN D ON ACRYLAMIDE INDUCED STRIATED MUSCLES DYSTROPHY OF RAT'S TONGUE (HISTOLOGICAL AND HISTOCHEMICAL STUDY)
}

\author{
Abd El-Hamied Salah Eldin El-Zarka*, Kamal Abd-Elrhaman Kamal ${ }^{* *}$, Mohamed Mahmoud Ahmed ${ }^{* * *}$
}

\begin{abstract}
Aim: The aim of the present study was be directed to evaluate therapeutic efficacy of vitamin D on acrylamide (ACR) induced striated muscles dystrophy of rat's tongue. Material and methods: Forty adult male albino rats were used as experimental animals and divided into four equal groups $(\mathrm{G}(\mathrm{s})$ ):10 animals in each one for two successive periods (5 animals for 20 days: GA) and (the other 5 animals for 40 days: GB). After termination of the experiment, the tongue Tissue sections were cut at $4 \mu \mathrm{m}$ for hematoxylin and eosin (H\&E) staining, another tissue section were cut at $3 \mu \mathrm{m}$ for staining with Phosphotungestic acid haematoxylin (PTAH). Results: Gross observation results of tongue mucosa in the various groups used, throughout the experimental periods, showed various changes compared to G1. The histomorphometric results revealed that PTAH staining has variability in the area percentage throughout the groups used. There were high significant differences between (G2A and G3A), and (G2B and G3B). In conclusions: Realization of striated muscle damage in rat's tongue by ACR which becomes worse with prolonged duration. Moreover vitamin D has a significant protective effect when compared with its therapeutic effect on ACR-induced striated muscle injury of rat's tongue.
\end{abstract}

\section{INTRODUCTION}

The muscle tissue is a primary site for glycogen storage, insulin uptake and amino acid catabolism, playing a crucial role in metabolism and heat production through the contraction of muscle fibers ${ }^{(1)}$, skeletal muscles tissue are under voluntary control and are responsible for movement and contraction of skeletal parts of the body ${ }^{(2)}$. Skeletal muscle contraction needs both $\mathrm{Ca} 2+$ and Adenosine triphosphate (ATP) ${ }^{(3)}$. Tongue is one of the highly skeletal muscular organs in the body ${ }^{(4)}$. Loss of muscle tissue observed in aging or in degenerative muscle diseases ${ }^{(5)}$. Degeneration may involve reversible muscle atrophy in response to situations like transient starvation or disuse that is manifested by shrinking of the myofiber often without loss of myonuclei ${ }^{(6)}$. Muscle fiber degeneration reflected by increased plasma levels of muscle proteins (i.e. creatine kinase, myosin heavy chain). It is triggered by release of calcium from SR, what drives calcium-dependent proteolysis leading to tissue degeneration ${ }^{(7)}$. A number of studies and reviews postulate oxidative stress as a major contributor of muscle atrophy ${ }^{(8,9)}$. Oxidative stress is a condition that the production and clearance of free radicals are unbalanced and that the clearance rate of free radicals is not sufficient to meet their production ${ }^{(10)}$. Some studies demonstrated that ACR induced cytotoxicity was relevant to oxidative stress $^{(11,12)}$. ACR is a low molecular weight, water soluble vinyl monomer from which polyacrylamides are synthesized to be used in the personal care and grooming products, such as lotions, cosmetics and deodorants ${ }^{(13)}$.

\footnotetext{
* Demonstrator of Oral and Dental Pathology, Faculty of Dental Medicine (Boys- Cairo), Al-Azhar University.

** Lecturer of Oral and Dental Pathology, Faculty of Dental Medicine (Boys- Cairo), Al-Azhar University.

*** Professor of Oral and Dental Pathology, Faculty of Dental Medicine (Boys- Cairo), Al-Azhar University.
} 
Direct exposure to ACR may result from ingestion of high carbohydrate foods prepared at high temperatures such as potato crisps, crackers and French fries ${ }^{(14)}$. Indirect exposure may result from residual traces of the monomer in food packaging where polyacrylamide is used as a binding agent ${ }^{(15)}$. ACR has been reported to be neurotoxic ${ }^{(16)}$ toxic to the reproductive system ${ }^{(17)}$ and carcinogenic in experimental animals ${ }^{(18)}$.

Skeletal muscle regeneration is clearly essential for long- lived humans where there is much opportunity for severe injury to occur during their life span ${ }^{(19)}$. Regeneration the extent of initial myofiber death depends on the type of injury: some may affect only a portion of the long multinucleated myofiber ${ }^{(20)}$. Muscle cells depend on efficiently repairing tears in their sarcolemma this is a Ca2-dependent process ${ }^{(21)}$. Cell membrane repair involves use of intracellular compartments, which in case of muscle cells include mitochondria, lysosomes ${ }^{(22)}$. A dietary antioxidant is a substance that significantly decreases the harmful effects of "reactive species", that disrupt normal physiological function on a cellular level in humans. Examples include the antioxidant nutrients vitamin $\mathrm{C}$, vitamin $\mathrm{E}$ and vitamin $\mathrm{D}^{(23)}$. A broad spectrum of cytokines, growth factors and vitamins is known to facilitate muscle regeneration during muscle injury and neuromuscular diseases. Vitamin D is a secosteroid that is produced in the skin and is synthesized in the liver and kidney in its biologically active form, 1.25-Dihydroxy-vitamin D. So far there is increasing evidence that Vitamin D positively affects the muscle function as well as the muscle force ${ }^{(24)}$. Beyond its critical function in calcium homeostasis, vitamin D has recently been found to play an important role in the modulation of the immune/inflammation system via regulating the production of inflammatory cytokines and inhibiting the proliferation of proinflammatory cells, both of which are crucial for the pathogenesis of inflammatory diseases ${ }^{(25)}$. Recent studies indicate that vitamin $\mathrm{D}$ can regulate the adaptive immune response in various inflammatory and autoimmune diseases $^{(26,27)}$. In this regard, the ultimate goal of the current study is directed to investigate the effect of vitamin D on ACR-induced striated muscle dystrophy of rat's tongue.

\section{MATERIAL AND METHODS}

I- Animal models: Forty adult male albino rats weighting (200-250 g) ,the procedures were conducted in accordance with the committee for the purpose of control and supervision on experiments on animals (CPCSEA Guidelines) (28).

II- Experimental design: After a week of adaptation, the animals were divided into four equal groups $(\mathrm{G}(\mathrm{s}))$ (10 animals in each one) for two successive periods ( 5 animals for 20 days: GA) and (the other 5 animals for 40 days: GB) as follow: (G1), negative control group: animals were fed with standard diet, without receiving any kind of treatment and considered as negative control group. (G2), positive control group: animals were received $15 \mathrm{mg} / \mathrm{kg}$ body weight of (ACR) orally once daily by gastric tube. (G3), chemoprevention group: animals were received $15 \mathrm{mg} / \mathrm{kg}$ body weight of ACR orally once daily, in addition; they were received, vitamin D $(8.3 \mathrm{mg} / \mathrm{kg})$ intraperitoneally ${ }^{(30)}$ one week before, as well as during application of ACR on alternative days group.(G4), therapeutic group: animals were received $15 \mathrm{mg} / \mathrm{kg}$ body weight of ACR orally once daily, in addition; they were received, vitamin $\mathrm{D}(8.3 \mathrm{mg} / \mathrm{kg})$ intraperitoneally one, three and seven days after application of ACR ${ }^{(31)}$.

III-Chemicals used: 1 -ACR (99.9\% purity) and Vitamin D3 were purchased from Sigma Aldrich chemical Company (Egypt).

IV-Sample collection and preparation: All animals in this study were euthanized after each 
period of the investigation. Then the tongue tissues were excised fixed in $10 \%$ neutral buffered formalin, and routinely processed to be examined microscopically.

\section{V- Preparation for histological examination:}

Tissue sections were cut at $4 \mu \mathrm{m}$ for H\&E staining for examination and recording the histological findings. Another tissue sections were cut at $3 \mu \mathrm{m}$ for staining with (PTAH). The tissue sections were examined using light microscope to assess the prevalence of localization of PTAH within the tissues. In addition, image analysis computer system was used to assess area percentage of PTAH staining. The image analysis was performed using Leica QWIN V3 image analyzer computer system.

VII- Statistical analysis: The comparison between two independent groups with quantitative data and parametric distribution was done by using independent t-test. The comparison between more than two independent groups with quantitative data and parametric distribution was done by using one way analysis of variance (ANOVA), the p-value reference was as the following: $\mathrm{P}>0.05$ : Non significant, $\mathrm{P}<0.05$ : Significant, $\mathrm{P}<0.01$ : Highly significant.

\section{RESULTS}

Gross observation of tongue mucosa of all animals in G1 showed no observable abnormalities(Plate 1-Fig.1) (arrow), G2A showed diffuse swelling and some areas of deep redness (Plate-1Fig.2)(arrow), G2B showed diffuse swelling, areas of deep redness and bleeding(Plate-1Fig.3) (arrow), G3A showed localized swelling with some areas of redness(Plate-1Fig.4) (arrow), G3B showed localized swelling and some areas of deep redness(Plate1Fig.5) (arrow), G4A showed diffuse swelling and some areas of deep redness(Plate-1Fig.6) (arrow), and G4B showed diffuse swelling and multiple areas of redness(Plate-1Fig.7) (arrow).

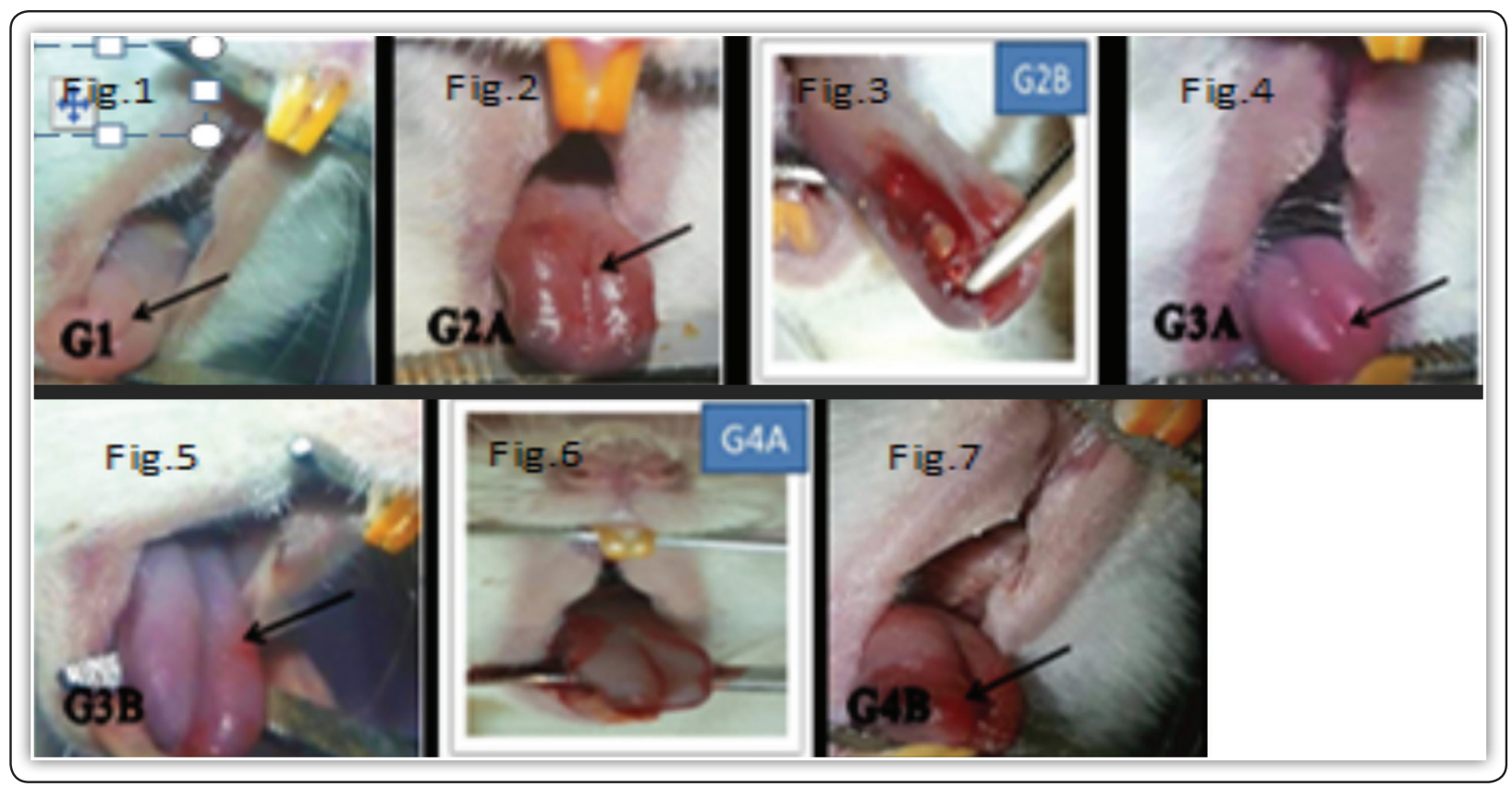

Plate (1) Showing gross observation results of tongue mucosa in the various groups used, throughout the experimental periods, showed various changes compared to G1 


\section{Histopathological and histochemical results:}

Histological results: $H \& E$ stain in tongue tissue section showed G1 those that the nuclei were multiple, vesicular, elongated and peripheral in position under the sarcolemma(arrows) (Plate:2-Fig.1), G2A showed fragmentation of the sarcoplasm (arrow A) and vasodilation of blood vessels (arrow B) (Plate:2-Fig.3), G2B showed mesenchymal tissue infiltrated by inflammatory cells (arrow) (Plate:2-Fig .5), G3A showed abnormal wavy course of muscle fibers with limited areas of the spaces(arrow) (Plate:2-Fig.7), G3B showed those are dense pyknotic nuclei (arrow A) with variable sized spaces between muscle fibers(arrow B) (Plate:2-Fig.9), G4A showed abnormal wavy course of muscle fibers(Plate:2-Fig.11) (arrows), G4B showed variable sized spaces between muscle fibers(arrows (Plate:2-Fig.13). H\&E stain (x400). Histochemical results: PTAH stain in tongue tissue section showed G1 closely packed uniform pattern of parallel bundles of striated muscle fibers (bluish color) (arrow A) and elastic fibers appeared pale brownish red between the muscle bundles (arrow B) (Plate:2-Fig.2), G2A showed splitting of the myofibers and fragmentation of the sarcoplasm (arrow A), and limited areas of brownish elastic fibers (arrow B) (Plate:2-Fig.4), G2B showed a separation of muscle fibers were seen (arrow) (Plate:2-Fig.6), G3A showed limited narrow spaces between the muscle fibers (arrow) (Plate:2-Fig.8), G3B showed deeply stained peripherally located nuclei (arrow ) (Plate:2-Fig.10), G4A showed limited narrow spaces between the muscle fibers (arrow) (Plate:2-Fig.12), G4B showed fragmentation of the sarcoplasm (arrow) (Plate:2-Fig.14), PTAH stain (x400).

\section{Statistical analysis results:}

PTAH staining at 20 days: there was high significant difference between $\mathrm{G} 1$ and (G2A, G3A and G4A), also between G3A and (G2A and G4A) where $\mathrm{P}<0.01$, and there was no significant difference between $\mathrm{G} 2 \mathrm{~A}$ and G4A where $\mathrm{P}$ value was 0.093 . PTAH staining at 40 days: there was high significant difference between G1 and (G2B , G3B and G4B), also between G3B and (G2B and $\mathrm{G} 4 \mathrm{~B}$ ) where $\mathrm{P}<0.01$, also there was no significant difference between (G2B and G4B) where P $>0.05$ (Chart.1).

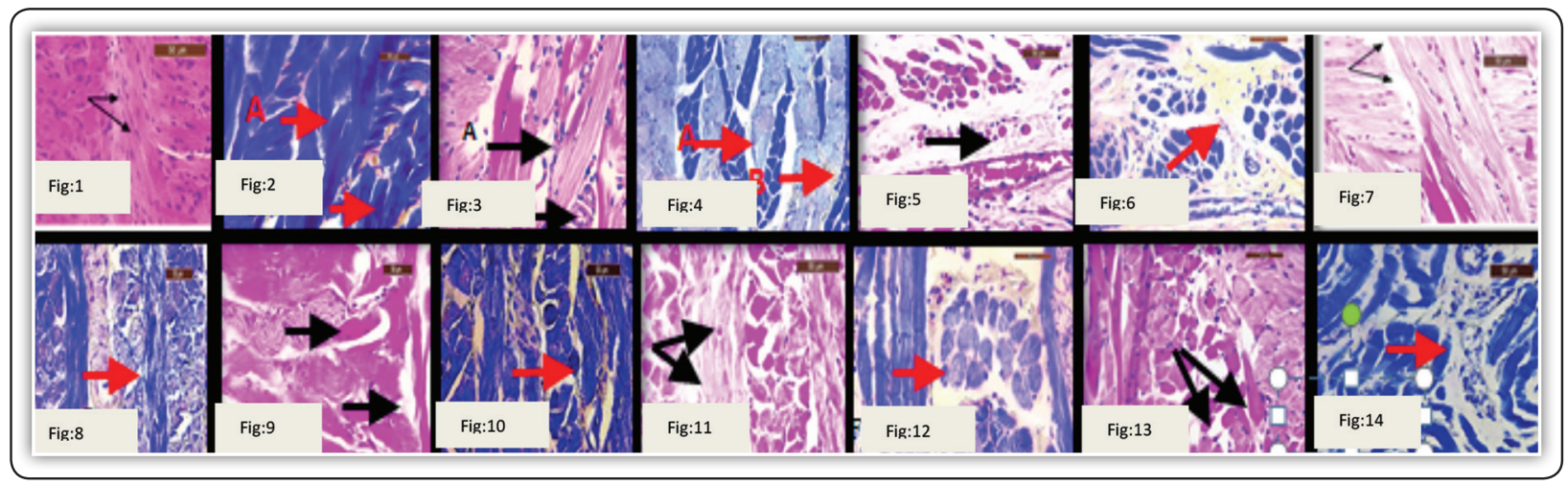

Plate (2) Showing histopathological (H\&E) and histochemical (PTAH) results showed various changes compared to G1 


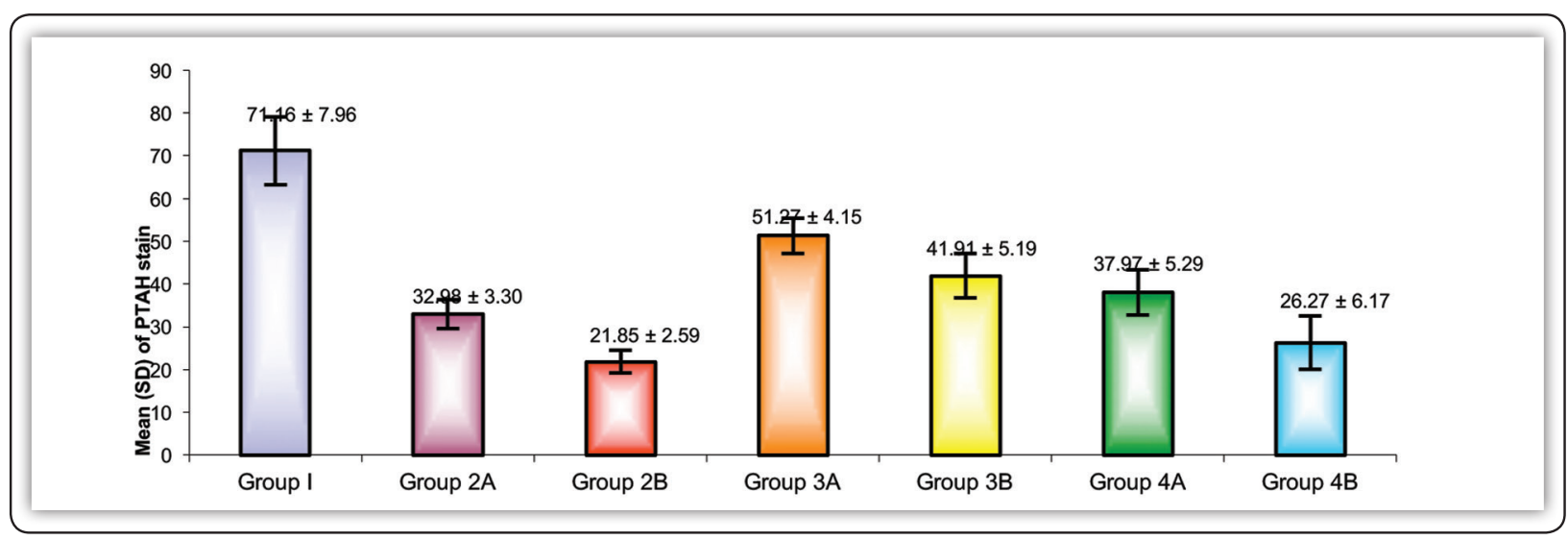

Chart (1) Representing mean area \% results in all groups at 20 and 40 days regarding PTAH stain

\section{DISCUSSION}

In the current study, the effects of ACR on the striated muscle fibers of the adult male albino rats tongue showed variable changes in both gross finding as well as on submicroscopic observation when utilizing either H\&E stain or PTAH stain. In the present study, G2A the deteriorations of muscle pattern with the presence of multiple spaces which could reflect a fatty degeneration between the muscle fibers and inflammatory cell infiltrates of the animals, similar histological features were observed by Weiss et al. ${ }^{(31)}$ they noticed that cross-sectional area of myofibers was decreased significantly in association with increased oxidative damage in addition to fatty infiltration. In the present study, PTAH stain analysis supported the deterioration of muscle fibers as there were High significant differences among the mean numbers of PTAH in G2A compared to G1 ( $\mathrm{p} \leq 0.01)$. The degenerative findings of the current study are mostly related to the oxidative stress effect of ACR on the striated muscle, this result coincides with the study of $\mathrm{Al}$ Serwi et al. ${ }^{(11)}$ who stated that destruction of muscle fibers with edema and inflammatory cell infiltration are prominent features of oxidative stress effect on skeletal muscle. In the current study, worse effect of ACR was more obvious in gross observation as well as on the histological features in animals of G2B those had received ACR for 40 days. Splitting of the myofibers was shown to be increased and fragmentation of the sarcoplasm in addition to infiltrated by huge number of inflammatory cells. These results were emphasized by PTAH stain analysis as there were high significant differences among PTAH expression in G2B as compared to G1and G2A ( $\mathrm{p} \leq 0.01$ ), which may be attributed to the worse degenerative effect on muscle fibers in G2B as compared to G2A and also may be due to the prolonged exposure to ACR. These results are in line with Al-Serwi et al. ${ }^{(11)}$, whom stated that chronic exposure to ACR might lead to skeletal muscle damage in rat tongue which becomes worth with prolonged duration of exposure. This results also was confirmed by Shinomol et al. ${ }^{(32)}$ as they stated that the toxic effects of ACR in different tissues is a dose dependent which increased by increasing the dose or the duration. The current study reflected the positive effect of vitamin D as chemopreventive measure on animals in G3A, also gross observations as well as the histological results revealed some alterations. There were high significant differences among the mean numbers of PTAH in G3A (51.27\%) as compared to G2A $(32.98 \%)$ and $p$ value $(\mathrm{p} \leq 0.01)$. These findings may be due to the protective effect of vitamin $\mathrm{D}$ against muscle degeneration. Another explanation vitamin D was shown to inhibit immune reactions in chronic inflammatory diseases, as reviewed by Hewison et $\mathrm{al}^{(33)}$. The fore mentioned results of the present study are consistent with the study by Vivian et al. ${ }^{(34)}$ 
on mice, who expressed that vitamin $\mathrm{D}$ administration reduces the number of activated macrophages and promotes a shift from pro-inflammatory cytokinesecreting macrophages to an amoeboid cell type, still capable of phagocytosis ${ }^{(34)}$. In the current study, the animals of G3B model had positive effect compared to those of G2A and G2B, which revealed negative effect was noticed when compared to G3A, also there were significant differences among the mean numbers of PTAH in G3A (51.27\%) as compared to G3B (41.91\%) and p value ( $\mathrm{p} \leq 0.05$ ), which may be attributed to the degenerative effect on muscle fibers in G3B as compared to G3A that may be due to toxicity results from prolong exposure to ACR. The histological results revealed that H\&E stain in G3B showed keratinized stratified regular acanthotic rete ridges and subepithelial mesenchymal tissue infiltrated by inflammatory cells. Irregular pattern of wavy course muscle fibers with variable sized spaces in between was seen. This could be due to the effect of vitamin D administration. The inhibition of the inflammatory reaction mediated by cytokines, which are secreted by macrophages, (Interleukin (IL)-1, IL-6 and tumor necrosis factor -1 (TNF $\alpha)$ ), may prevent muscle degeneration and maintain cellular heamostasis. These are in agreement with other researcher ${ }^{(35)}$. In the current study, the animals of G4A model, those having received vitamin $\mathrm{D}$, as therapeutic measure, intraperitoneally once daily for 3 times as follow: first day, third day and the fifth day revealed low positive effect compared to those of G3A\&G3B. Also, there were high significant differences among the mean of the area percentage of PTAH stain in animals in G4A (37.97\%) as compared to those in G3A (51.27\%) and $\mathrm{p}$ value was $(\mathrm{p} \leq 0.01)$. These result suggested that massive effect of vitamin $\mathrm{D}$ in chemoprevention rather than treatment of muscle degeneration. In the present study, similarly, animals in G4B model revealed its positive effect compared to those of $\mathrm{G} 2 \mathrm{~A} \& \mathrm{~B}$. Contrarily, a relative positive effect was noticed when comparing with G3 and G4.
Also, there were high significant differences among the mean numbers of PTAH stain distribution in G4B (26.27\%) when compared to G3B (41.91\%) (p $\leq 0.01)$. In the present study the treatment effect of vitamin $\mathrm{D}$ was after 5 day of its application. Barker el al. ${ }^{(30)}$ reported that uses vitamin D $8.3 \mathrm{mg} / \mathrm{kg}$ once a day for 14 days have anti-nociceptive properties which was accompanied by antioxidant effects and inhibition of microglia activity. These results may be related to the fact that regenerative effect of vitamin $\mathrm{D}$ may require long time of treatment.

\section{CONCLUSIONS}

1. Realization of striated muscle damage in rat's tongue by ACR which becomes worth with prolonged duration.

2. A significant efficacy of Vit D as protective and regenerative effect when compared with its therapeutic effect on ACR-induced striated muscle injury of rat's tongue.

\section{REFERENCES}

1. Scaramozza A. The regulation of satellite cells during skeletal muscle regeneration and neuromuscular disease. Alma. 2012: 2-94.

2. Mayer L, Bhikha R. Relationship between tissues of the body with tibb.A Science of Medicine, The Art Of Care, Tibb Institue. 2016:1-26.

3. Russell AP, Foletta VC, Snow RJ, Wadley GD. Skeletal muscle mitochondria: a major player in exercise, health and disease. Biochimica et biophysica acta (BBA)-general subjects. 2014; 1840:1276-84.

4. Krönert H, Pleschka K. Lingual blood flow and its hypothalamic control in the dog during panting. Pflügers $\mathrm{Ar}-$ chiv. 1976;367:25-31.

5. Zanou N, Gailly P. Skeletal muscle hypertrophy and regeneration: interplay between the myogenic regulatory factors (MRFs) and insulin-like growth factors (IGFs) pathways. Cellular and Molecular Life Sciences. 2013;70:4117-30.

6. Rai M, Nongthomba U, Grounds MD. Skeletal muscle degeneration and regeneration in mice and flies. Current topics in developmental biology. 2014;108:247-81. 
7. Yin H, Price F, Rudnicki MA. Satellite cells and the muscle stem cell niche. Physiological reviews. 2013;93:23-67.

8. Avin KG, Chen NX, Organ JM, Zarse C, O’Neill K, Conway RG,et al. Skeletal muscle regeneration and oxidative stress are altered in chronic kidney disease. PloS one. 2016; 11:1-15.

9. Powers SK, Kavazis AN, DeRuisseau KC. Mechanisms of disuse muscle atrophy: role of oxidative stress. Physiology, Regulatory, Integrative and Comparative Physiology. 2005; 288: 337- 44

10. Halliwell B, Cross CE. Oxygen-derived species: their relation to human disease and environmental stress. Environmental Health Perspectives. 1994;102:5-12..

11. Al-Serwi RH, Ghoneim FM. The impact of vitamin E against acrylamide induced toxicity on skeletal muscles of adult male albino rat tongue: Light and electron microscopic study. Microscopy and Ultrastructure. 2015; 3:137- 47.

12. Rodríguez-Ramiro I, Ramos S, Bravo L, Goya L, Martín MÁ. Procyanidin B2 and a cocoa polyphenolic extract inhibit acrylamide-induced apoptosis in human Caco-2 cells by preventing oxidative stress and activation of JNK pathway. Nutritional Biochemistry. 2011;22:1186-94.

13. Exon J. A review of the toxicology of acrylamide. Toxicology and Environmental Health, Part B. 2006;9:397-412.

14. Tareke E, Rydberg P, Karlsson P, Eriksson S, Törnqvist M. Analysis of acrylamide, a carcinogen formed in heated food stuffs. Agricultural and Food Chemistry. 2002;50:4998-5006

15. Zhang Y, Zhang G, Zhang Y. Occurrence and analytical methods of acrylamide in heat-treated foods: Review and Recent Developments. A. 2005;1075:1-21.

16. Seale SM, Feng Q, Agarwal AK, El-Alfy AT. Neurobehavioral and transcriptional effects of acrylamide in juvenile rats. Pharmacology Biochemistry and Behavior. 2012;101:77-84

17. Ma Y, Shi J, Zheng M, Liu J, Tian S, He X, et al. Toxicological effects of acrylamide on the reproductive system of weaning male rats. Toxicology and Industrial Health. 2011;27:617-27

18. Hogervorst JG, Baars B-J, Schouten LJ, Konings EJ, Goldbohm RA, van den Brandt PA. The carcinogenicity of dietary acrylamide intake: a comparative discussion of epidemiological and experimental animal research. Critical Reviews in Toxicology. 2010;40:485-512.
19. Relaix F, Zammit Ps. Satellite cells are essential for skeletal muscle regeneration: the cell on the edge returns centre stage. Development. 2012;139:2845-56.

20. Rai M, Nongthomba U, Grounds MD. Skeletal muscle degeneration and regeneration in mice and flies. Current topics in developmental biology. 2014;108:247-81.

21. McNeil PL, Steinhardt RA. Plasma membrane disruption: repair, prevention, adaptation. Annual Review of Cell and Developmental Biology. 2003;19:697-731.

22. Sharma N, Medikayala S, Defour A, Rayavarapu S, Brown KJ, Hathout Y, et al. Use of quantitative membrane proteomics identifies a novel role of mitochondria in healing injured muscles. Biological Chemistry. 2012;287: 30455-67.

23. Adams AK, Wermuth EO, Mcbride PE. Antioxidant vitamins and the prevention of coronary heart disease. American Family Physician. 1999;60:895-906.

24. Pfeifer M, Begerow B, Minne H. Vitamin D and muscle function. Osteoporosis International. 2002;13:187-94.

25. Yin K, Agrawal DK. Vitamin D and inflammatory diseases. Inflammation Research. 2014;7:69-87.

26. Tiosano D, Wildbaum G, Gepstein V, Verbitsky O, Weisman Y, Karin N, et al. The role of vitamin d receptor in innate and adaptive immunity: A study in hereditary vitamin d-resistant rickets patients. Clinical Endocrinology and Metabolism. 2013;98:1685-93.

27. Olliver M, Spelmink L, Hiew J, Meyer-Hoffert U, Henriques-Normark B, Bergman P. Immunomodulatory effects of vitamin $\mathrm{D}$ on innate and adaptive immune responses to Streptococcus pneumoniae. Infectious Diseases. 2013; 208:1474-81.

28. Pereira S, Tettamanii M. Ahimsa and alternatives-the concept of the 4th R. The CPCSEAin India. Altex. 2005; 22:3-6.

29. Stratos I, Li Z, Herlyn P, Rotter R, Behrendt A-K, Mittlmeier T, et al. Vitamin D increases cellular turnover and functionally restores the skeletal muscle after crush injury in rats. Pathology. 2013;182:895-904.

30. Barker T, Schneider ED, Dixon BM, Henriksen VT, Weaver LK. Supplemental vitamin D enhances the recovery in peak isometric force shortly after intense exercise. Nutrition and Metabolism. 2013; 69:1-10.

31. Weiss DJ, Casale GP, Koutakis P, Nella AA, Swanson SA Zhu Z, et al. Oxidative damage and myofiber degeneration in the gastrocnemius of patients with peripheral arterial disease. Translational Medicine. 2013;11:2-9. 
32. Kunnel Shinomol G, Raghunath N, Mukunda Srinivas Bharath M. Prophylaxis with Bacopa monnieri attenuates acrylamide induced neurotoxicity and oxidative damage via elevated antioxidant function. Central Nervous System Agents in Medicinal Chemistry (Formerly Current Medicinal Chemistry-Central Nervous System Agents). 2013;13:3-12.

33. Hewison M. Vitamin D and the immune system: new perspectives on an old theme. Endocrinology and Metabolism Clinics. 2010;39:365-79.
34. Lee V, Rekhi E, Kam JH, Jeffery G. Vitamin D rejuvenates aging eyes by reducing inflammation, clearing amyloid beta and improving visual function. Neurobiology of Aging. 2012;33:2382-89.

35. Wang J, Ohno-Matsui K, Yoshida T, Shimada N, Ichinose $\mathrm{S}$, Sato T, et al. Amyloid- $\beta$ up-regulates complement factor B in retinal pigment epithelial cells through cytokines released from recruited macrophages/microglia: Another mechanism of complement activation in age-related macular degeneration. Cellular Physiology. 2009;220:119-28. 